\title{
The Role of Nanobiotechnology in the Development of Personalized Medicine
}

\author{
K.K. Jain \\ Jain PharmaBiotech, Basel, Switzerland
}

Personalized medicine is defined as the prescription of specific treatments best suited for an individual taking into consideration both genetic and other factors that influence the response to therapy [1]. Personalized medicine is the best approach to integrate new biotechnologies into medicine in order to improve understanding of the pathomechanism of diseases, molecular diagnosis and integration with therapeutics. Nanomedicine is defined as the application of nanobiotechnology to medicine [2]. Nanobiotechnology also makes important contributions to personalized medicine through refinement of various technologies used for diagnostics and therapeutics as well as interactions among these, as shown in figure 1 [3].

Nanobiotechnologies provide refinements in drug discovery and formulations for targeted drug delivery. Molecular biomarkers, defined as cellular alterations to a cell at DNA, RNA, metabolite or protein levels, serve as common reference points for diagnosis and therapeutics [4]. Biomarkers have been discovered by nanobiotechnology-based approaches and not only serve as indicators of various diseases, but also guide therapeutics, thus enabling personalization of treatment. Single molecules of biomarkers, bacteria and viruses have been detected by nanobiotechnology-based assays, and they extend the

\section{KARGER}

Fax +4161306 1234

E-Mail karger@karger.ch

www.karger.com
(C) 2010 S. Karger AG, Basel

1011-7571/11/0201-0001\$38.00/0

Accessible online at:

www.karger.com/mpp limits of diagnostics for presymptomatic diagnosis and preemptive treatment of some diseases.

Nanobiotechnology techniques provide an analysis of DNA and protein and could dramatically improve speed, accuracy and sensitivity compared to conventional molecular diagnostic methods [5]. Nanobiotechnologybased approaches can provide more precise measurements and insight into nanoscale inherent functional components of living cells. Nanobiotechnology facilitates point-of-care diagnosis and the integration of diagnostics with therapeutics, thus advancing the development of personalized medicine.

Molecular imaging requires an accumulation of contrast agent in the target site, often most efficiently achieved by steering nanoparticles containing contrast agent to the target. For imaging modalities with low sensitivity, nanoparticles bearing multiple contrast groups provide signal amplification. The same nanoparticles can deliver both contrast medium and drug, allowing monitoring of biodistribution and therapeutic activity simultaneously. Nanoparticles share functions such as target recognition and binding with many subcellular organelles such as ribosomes, proteasomes, ion channels and transport vesicles which are of similar sizes. The materials used to synthesize nanoparticles include natural proteins and poly- 
Fig. 1. Relationship of nanobiotechnology

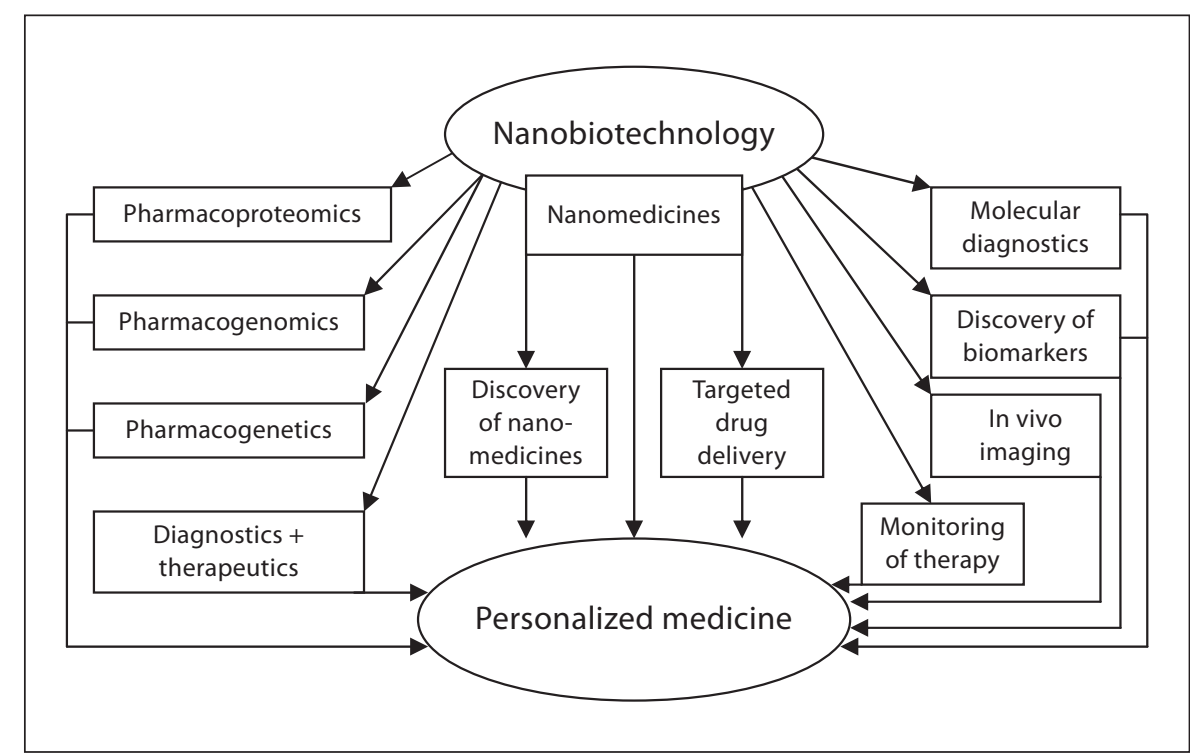
and personalized medicine.

mers, artificial polymers, dendrimers, fullerenes and other carbon-based structures, lipid-water micelles, viral capsids, metals, metal oxides and ceramics. Signal generators incorporated into nanoparticles include iron oxide, gadolinium, fluorine, iodine, bismuth, radionuclides, quantum dots and metal nanoclusters [6].

Nanoparticulate agents such as superparamagnetic contrast agent and perfluorocarbon nanoparticles are being intensively studied as novel tools for molecular imaging that will enable noninvasive characterization and segmentation of patients to allow personalized therapy. Multifunctionality is the key advantage of nanoplatforms over traditional approaches as they have been employed in several biomedical imaging modalities such as optical imaging, computed tomography, ultrasound, magnetic resonance imaging, single photon emission computed tomography and positron emission tomography [7]. Targeting ligands, imaging labels, therapeutic drugs and many other agents can all be integrated into the nanoplatform to allow targeted molecular imaging and personalized medicine.

Several nanotechnologies, including nanoparticles and nanodevices such as nanobiosensors and nanobiochips, are used to improve drug discovery and development. Some of the currently used medicines are being personalized, but the discovery and development of some of the new medicines will take personalization into consideration at an early stage. Nanobiotechnology already makes a significant contribution to drug discovery [8].
Drug delivery is an important part of modern pharmaceuticals. Targeted delivery to the desired organ/site of action and passage through biological barriers are important for the safe and effective use of drugs. Conjugation of nanocarriers to specific ligands and to aptamers allows specific targeting with improved clinical efficacy [9]. Nanoparticle-based improvements in drug delivery will enhance personalized medicine. Nanotechnology has also made important contributions to refinements in the delivery of biological therapies such as cell and gene therapies, which are suitable for personalized treatments.

An example of the use of nanotechnology in personalizing treatment is the combination of nanoparticle-based imaging and therapy in cancer [10]. Several nanocomposite materials with synergistic components for diagnosis and treatment have been translated into the clinical management of cancer [11]. Among a selection of diagnostic and therapeutic components, the incorporation of immune target specificity by nanostructures introduces a new type of treatment modality, nanoimmunochemotherapy, for patients with cancer [12].

In conclusion, nanobiotechnologies facilitate the development of personalized medicine by extending the limits of diagnostics and facilitating the integration of diagnostics and therapeutics. Nanoparticle-based targeted therapy further improves the specificity of drugs and increases safety by reducing adverse effects. 


\section{References}

1 Jain KK: Textbook of Personalized Medicine. New York, Springer, 2009.

2 Jain KK: A Handbook of Nanomedicine. Totowa, Humana, 2008

> Jain KK: Nanomedicine: application of nanobiology in medical practice. Med Princ Pract 2008;17:89-101.

4 Jain KK: A Handbook of Biomarkers. New York, Springer, 2010.

5 Jain KK: Applications of nanobiotechnology in clinical diagnostics. Clin Chem 2007;53: 2002-2009.
6 Debbage P, Jaschke W: Molecular imaging with nanoparticles: giant roles for dwarf actors. J Histochem Cell Biol 2008;130:845875.

7 Cai W, Chen X: Nanoplatforms for targeted molecular imaging in living subjects. Small 2007;3:1840-1854.

8 Jain KK: Nanotechnology in drug discovery; in Seethala R (ed): Handbook of Drug Screening, ed 2. New York, Informa, 2009, pp 465-476.

-9 Debbage P: Targeted drugs and nanomedicine: present and future. Curr Pharm Des 2009;15:153-172.

10 Jain KK: Application of nanobiotechnology in cancer therapeutics; in Lu Y, Mahato RI (eds): Pharmaceutical Perspectives of Cancer Therapeutics. New York, Springer, 2009, pp 245-268.
1 Khemtong C, Kessinger CW, Gao J: Polymeric nanomedicine for cancer MR imaging and drug delivery. Chem Commun (Camb) 2009; 24:3497-3510.

12 Kateb B, Chiu K, Black KL, Yamamoto V, Khalsa B, Ljubimova JY, Ding H, Patil R, Portilla-Arias JA, Modo M, Moore DF, Farahani K, Okun MS, Prakash N, Neman J, Ahdoot D, Grundfest W, Nikzad S, Heiss JD: Nanoplatforms for constructing new approaches to cancer treatment, imaging, and drug delivery: what should be the policy? Neuroimage 2010, E-pub ahead of print. 